\title{
Beyond Bonferroni: less conservative analyses for conservation genetics
}

\author{
Shawn R. Narum
}

Published online: 8 August 2006

(C) Springer Science+Business Media B.V. 2006

\section{Erratum to: Conserv Genet \\ DOI: 10.1007/s10592-005-9056-y}

In the example for the B-H FDR procedure, four incorrectly printed values create confusion for the reader. Corrections in the Methods section, second paragraph, third and fourth sentences: "The first $p$-value to satisfy $p_{i} \leq i / k \times \alpha$ is $p_{(10)}$ since $p_{(10)}=$ $0.032 \leq 10 / 15 \times 0.05=0.0333$. Thus pairwise tests in the experiment with $p$-values less than or equal to 0.0333 reject the null hypothesis. The gain in power

with the B-H method FDR over the Bonferroni procedure (critical values of 0.033 and 0.003 respectively in this example) are substantial." Corrections in the Methods section, last paragraph, last sentence: "This critical value is intermediate relative to those calculated from Bonferroni (0.0033) and B-H method FDR (0.0333)."

The online version of the original article can be found at http:// www.dx.doi.org/10.1007/s10592-005-9056-y

S. R. Narum $(\bowtie)$

Columbia River Inter-Tribal Fish Commission, 3059-F

National Fish Hatchery Road, Hagerman, ID 83332, USA

e-mail: nars@critfc.org 